\title{
O Ensino de Programação na Educação Básica: Uma Revisão da Literatura
}

\author{
Franciely Alves de Souza, Taciana Pontual Falcão, Rafael Ferreira Mello \\ Universidade Federal Rural de Pernambuco (UFRPE) - Recife - PE - Brasil \\ francielyalves835@gmail.com, \{taciana.pontual, rafael.mello\}@ufrpe.br
}

\begin{abstract}
Programming learning involves understanding a number of theories and practices, which enable the development of various skills. This article aims to present the result of a literature review regarding the methodologies used and tools that assist in teaching programming for children in a playful way, and emphasizing their positive contributions to basic education and interdisciplinary teaching, with students being able to solve different problems though computational concepts.
\end{abstract}

Resumo. A aprendizagem de programação envolve a compreensão de uma série de teorias e práticas, que possibilitam o desenvolvimento de diversas habilidades. Este artigo tem como objetivo apresentar o resultado de uma revisão da literatura referente a metodologias utilizadas e ferramentas que auxiliam no ensino de programação para crianças de forma lúdica, e enfatizando suas contribuições positivas para a educação básica e o ensino interdisciplinar, onde os alunos se tornam aptos a resolver diferentes problemas por meio de conceitos computacionais.

\section{Introdução}

Observando o atual cenário, são nítidas as dificuldades enfrentadas durante o processo de ensino e aprendizagem de programação, incluindo: método de ensino utilizado pelos professores, complexidade da lógica de programação e falta de vivência com determinados conceitos (Souza, 2016). Medeiros et al. (2020) citam também problemas de infraestrutura como laboratório com poucos computadores ou sem acesso a internet. Já Santos et al. (2006) destacam a falta de motivação dos estudantes, que pode ocorrer devido às suas dificuldades no aprendizado, até a metodologia de ensino adotada pelo professor em sala de aula.

A educação tem passado por várias mudanças de paradigmas e as aulas expositivas já não produzem o mesmo efeito no aprendizado (An et al., 2013). Principalmente quando se fala de programação, pois de acordo com Arimoto et al. (2019), o processo de aprendizagem de programação não é trivial, uma vez que os conceitos abordados e a carga de conhecimentos agregados não são simples e de fácil compreensão, especialmente para os alunos iniciantes. A partir disso, novas metodologias e estratégias de ensino precisam ser adotadas para proporcionar um ensino e aprendizagem de programação mais consistente e atraente para os alunos, inclusive com a inserção da programação na educação básica, na intenção que o aluno tenha acesso a conteúdos da computação na perspectiva de utilizar o computador como uma ferramenta para o desenvolvimento de sua criatividade, raciocínio lógico e capacidade de resolução de problemas.

O ensino de programação na educação básica é primordial para que as crianças e jovens possam desenvolver a habilidade de identificar e solucionar problemas, com criatividade e eficiência. Oro et al. (2015) ressaltam que o ensino de programação de 
computadores representa uma alternativa poderosa na qualificação da formação básica das crianças, contribuindo potencialmente para o processo de ensino e aprendizagem em todas as áreas do conhecimento.

Nesta perspectiva, de acordo com Ferri (2017), tem-se julgado pertinente investigar e propor o uso de plataformas de programação para alunos da Educação Básica focadas no desenvolvimento cognitivo. $\mathrm{O}$ ensino de programação favorece o desenvolvimento do raciocínio lógico e da capacidade de abstração, além de apoiar o desenvolvimento de habilidades como resolução de problemas e noções de causa e efeito (Gomes, 2015).

O ensino de programação na educação básica exige metodologias e estratégias que possibilitem um aprendizado lúdico e engajador, para que os alunos se motivem e sintam curiosidade de aprender novos conceitos. Para isso pode-se pensar na técnica de storytelling como uma estratégia para atrair a atenção e proporcionar um aprendizado diferenciado. Segundo Torres et al. (2008), o ato de contar histórias leva à diversão, estimula a imaginação e pode proporcionar um aumento no interesse do aluno pela aula.

A motivação deste trabalho é identificar quais são os objetivos do ensino de programação na educação básica e quais técnicas e ferramentas são utilizadas com maior frequência no ensino. Este trabalho está organizado da seguinte maneira: A Seção 2 apresenta os trabalhos relacionados. A Seção 3 descreve algumas ferramentas e estratégias utilizadas para o ensino de programação para crianças. A Seção 4 apresenta o método utilizado e as etapas da revisão da literatura em quatro subseções. A Seção 5 descreve os resultados obtidos após a análise em duas subseções. A Seção 6 detalha as considerações finais, limitações e trabalhos futuros.

\section{Trabalhos Relacionados}

Ferreira et al. (2021) apresentam uma revisão da literatura de trabalhos publicados de 2001 a 2019, que contém dados sobre o ensino de programação na educação básica com suporte do pensamento computacional e da ferramenta Scratch, com a finalidade de analisar experiências já vivenciadas e materiais didáticos já desenvolvidos para adaptá-los em trabalhos futuros a serem desenvolvidos em sala de aula, passando a utilizar a ferramenta Scratch para auxiliar no processo de resolver problemas de forma diferente.

Por sua vez, o trabalho de Silva (2017) apresenta uma busca de trabalhos publicados de 2012 a 2016, a fim de analisar quais estratégias de ensino são utilizadas com maior frequência para apoiar o ensino de programação na educação básica, com o intuito de tornar o ensino e aprendizagem lúdico e de fácil compreensão para os alunos. Como resultado, concluiu-se que existe uma preocupação sobre qual ambiente de programação utilizar para introduzir conteúdos de lógica de programação iniciais, de forma lúdica e engajadora.

Já Ferri et al. (2016) apresentam uma revisão sistemática da literatura a fim de analisar quais ferramentas são utilizadas no ensino de programação na educação básica, e quais são os benefícios do processo de ensino e aprendizagem de programação. Os autores obtiveram como resultados ferramentas que podem contribuir significativamente para apoiar a construção do conhecimento inicial, como Scratch e SuperLogo, tendo como objetivo desenvolver o pensamento lógico na intenção de melhorar o desempenho em outras disciplinas ainda na educação básica.

É importante destacar que, nos trabalhos citados nesta seção, é notável que existe uma busca significativa sobre quais ferramentas utilizar, e quais metodologias e estratégias de ensino aplicar. Ambos se assemelham com este artigo, no que se refere em obter respostas sobre como proporcionar um ensino e aprendizagem de programação que se aproxime da realidade dos alunos, com o diferencial de analisar se o pensamento computacional e seus conceitos podem contribuir para que conteúdos de outras disciplinas sejam abordados de forma lúdica facilitando o aprendizado. 


\section{Programação para Crianças}

Aprender a programar pode ser considerado como uma atividade complexa para qualquer pessoa iniciante, e essa ação se torna ainda mais difícil quando se trata de crianças. Quando estudam programação, as pessoas não só aprendem a programar, como também programam para aprender, noções de programação devem ser ensinadas desde cedo às crianças (Serrano, 2014). Ao ensinar programação para crianças, é importante utilizar ferramentas que possam dinamizar o processo de aprendizagem criativa. Um exemplo de ferramenta com interface voltada para crianças é o Scratch, um projeto do grupo Lifelong Kindergarten no Media $L a b$ do MIT (Instituto de Tecnologia de Massachusetts), idealizado por Mitchel Resnick. A ferramenta foi projetada para crianças, mas passou a ser utilizada por diferentes públicos, por se tratar de uma ferramenta prática e com diversas funcionalidades, trabalhando com programação baseada em blocos.

De acordo com Kelleher e Pausch (2005), a programação baseada em blocos procura abstrair a sintaxe de forma que os estudantes iniciantes mantenham seu foco apenas na semântica. Ela estimula o uso de estratégias para utilizar a computação criativa no ambiente educacional.

Programação em blocos funciona a partir da ação de arrastar e soltar componentes, que se caracterizam como blocos. Na construção de um programa, vários blocos são encaixados uns aos outros como um quebra-cabeça, levando em consideração que cada bloco possui encaixes específicos, já que as linguagens de programação em blocos possibilitam que o usuário consiga visualizar onde cada bloco pode ser encaixado (embora em alguns casos, a depender da ferramenta, seja possível encaixar blocos de forma errônea) (Weintrop et al., 2017; Kelleher e Pausch, 2005). A figura 1 mostra exemplos de blocos existentes na ferramenta Scratch, essa programação pode mudar a cor de botões através de um sensor, se o mouse estiver sobre o botão programado, ele mudará de cor, senão o botão terá sua cor padrão visível.

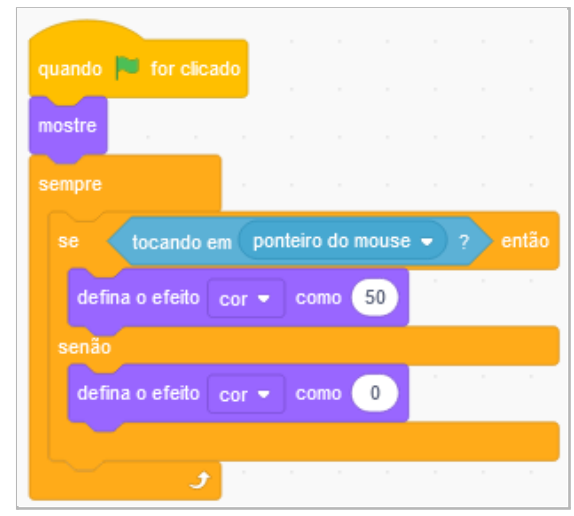

Figura 1: Programação de um sensor do mouse. Fonte: Scratch.

Além do Scratch, que se tornou uma das ferramentas mais utilizadas atualmente, é importante destacar a utilização do Scratch Jr., uma ferramenta voltada para crianças do ensino infantil, com idade de 5 a 7 anos, disponível em versão Android e IOS. O ensino de programação com o Scratch Jr. pode ser apoiado pela prática de storytelling, onde os alunos podem visualizar a descrição do problema a ser resolvido através de um contexto ilustrativo, na intenção de facilitar a compreensão. No contexto desse trabalho, storytelling é a contação de histórias com o auxílio de ferramentas digitais com o propósito de obter a atenção do público (Domingos, 2009; Palacios; Terenzo, 2016). O uso de storytelling no ensino e aprendizagem pode proporcionar momentos lúdicos e engajadores, onde a contrapartida para obter a solução de um problema é visualizar a história e abstrair tudo que não se faz necessário para a resolução de um determinado problema. 
De acordo com Oliveira et al. (2012), o storytelling tem benefícios como atrair o interesse dos alunos, transmitir o conteúdo de forma mais interessante, gerar uma comunicação mais próxima com o aluno, intertextualidade com outras disciplinas e garantir um aprendizado efetivo.

Uma habilidade que é bastante desenvolvida em programação para crianças é o pensamento computacional. De acordo com a visão de Wing (2006), o pensamento computacional é o processo de reconhecer aspectos da computação no mundo que nos rodeia e aplicar ferramentas e técnicas para entender e raciocinar sobre sistemas e processos naturais, sociais e artificiais. O pensamento computacional, no âmbito escolar, possibilita que os alunos solucionem problemas por partes, abstraiam o que não for necessário para a conclusão de uma tarefa, saibam reconhecer padrões existentes em diferentes problemas e criem algoritmos para solucioná-los. Segundo Selby e Woollard (2013), o pensamento computacional é um processo cognitivo ou de pensamento que envolve o raciocínio lógico pelo qual os problemas são resolvidos e os artefatos, procedimentos e sistemas são melhor compreendidos.

\section{Método}

Essa pesquisa é de caráter exploratório, baseada no protocolo proposto por Kitchenham e Charters (2007), sendo uma revisão da literatura, pautada na: 1) elaboração de perguntas de pesquisa; 2) definição das strings de busca; 3) definição de critérios de inclusão e exclusão dos trabalhos encontrados; 4) leitura dos artigos selecionados; e 5) apresentação e análise dos resultados. As subseções a seguir descrevem de forma detalhada cada etapa.

\subsection{Questões de Pesquisa}

Existem diversos trabalhos que promovem o ensino de programação na educação básica. No intuito de ter uma visão abrangente e compreender melhor as abordagens, técnicas e ferramentas mais utilizadas, foram organizadas as seguintes questões de pesquisa:

- QP1: Quais técnicas são utilizadas para propor o ensino de programação para crianças?

- QP2: Quais são os objetivos do ensino de programação para crianças?

- QP3: Quais ferramentas educacionais são utilizadas?

- QP4: Como o pensamento computacional influencia no desenvolvimento de conceitos de outras disciplinas?

\subsection{Busca de artigos}

Para esta revisão da literatura foi feita uma busca manual de trabalhos na literatura em português, nos anais dos seguintes eventos nacionais e internacionais:

- Simpósio Brasileiro de Informática na Educação (SBIE);

- Workshop de Informática na Escola (WIE);

- Congresso sobre Tecnologias na Educação (Ctrl+E);

- Simpósio Brasileiro de Jogos e Entretenimento Digital (SBgames);

- Ciclo de Palestras sobre Novas Tecnologias na Educação (CINTED);

- Conferência Internacional sobre Informática na Educação (TISE);

- Mostra de Práticas de Informática na Educação (MPIE);

- Workshop de Ensino em Pensamento Computacional, Algoritmos e Programação (WAlgProp);

Para realizar a busca, foram definidos diferentes grupos de palavras que têm relação com contexto de ensino, metodologia e idade dos alunos. No fim, os seguintes conjuntos de palavras-chave foram utilizados: 
- programação, escola, ensino fundamental;

- aprendizado, storytelling, lúdico;

- pensamento computacional, gamificação, robótica;

Após definir as palavras-chave, construímos uma string de busca usando os operadores lógicos (OR) e (AND) da seguinte forma:

1 "programação" AND “escola” AND "ensino fundamental”

2 "storytelling"

3 "aprendizado" AND "lúdico";

4 "pensamento computacional"

5 "gamificação" OR "robótica" OR (5)).

A combinação final da string de pesquisa usada foi: ((1) AND (2 AND 3) AND (4 )

\subsection{Critérios de inclusão e exclusão}

A seleção dos trabalhos foi conduzida em três etapas, sendo: (1) leitura do título e resumo; (2) leitura da introdução e conclusão; (3) leitura completa dos trabalhos e extração dos dados.

A inclusão dos trabalhos foi determinada de acordo com a relevância em relação às questões levantadas. Os critérios de exclusão foram definidos para eliminar os trabalhos que não disponibilizam acesso completo ao texto e que não disponibilizam acesso offline, todos eles estão detalhados na Tabela 1.

Tabela 1 - Critérios de Inclusão e exclusão.

\begin{tabular}{|l|l|}
\hline \multicolumn{1}{|c|}{ Critérios de Inclusão } & \multicolumn{1}{c|}{ Critérios de exclusão } \\
\hline $\begin{array}{l}\text { - Responder ao menos uma das perguntas de } \\
\text { pesquisa. }\end{array}$ & $\begin{array}{l}\text { - Trabalhos que não estão disponíveis para } \\
\text { downloads. }\end{array}$ \\
\hline - Artigos em português. & $\begin{array}{l}\text { - Trabalhos incompletos, tais como pôsteres e } \\
\text { apresentações; }\end{array}$ \\
\hline $\begin{array}{l}\text { - Artigos que tratam do uso de ferramentas, } \\
\text { programias ou metodologias para ensino de }\end{array}$ & \\
\hline - Publicações entre 2016 e 2020. & \\
\hline
\end{tabular}

\subsection{Extração dos dados}

Os dados foram extraídos e organizados em uma planilha ${ }^{1}$, contendo as seguintes informações: título do artigo, autores, instituição dos autores, ano de publicação, fonte da publicação, ferramenta utilizada, tipo de estudo (experimental, estudo de caso, intervenção), dados utilizados para gerar as análises, metodologia, visão geral do estudo, e respostas às perguntas de pesquisa. A Seção 5 descreve os resultados gerados após a síntese dos dados.

\section{Resultados: Análise e Discussão}

Nesta seção, os dados obtidos são analisados com foco em responder às questões de pesquisa previamente estabelecidas.

\footnotetext{
${ }^{1}$ encurtador.com.br/bkM14
} 


\subsection{Síntese dos dados}

A busca foi realizada no ano de 2020. A quantidade de trabalhos selecionados nos respectivos anais estão detalhados na tabela 2 .

Tabela 2. Total de artigos selecionados por base.

\begin{tabular}{|c|c|c|c|c|c|c|c|c|}
\hline \multicolumn{1}{|c|}{ Eventos e Quantidade de Artigos } \\
\hline WAlgProg & TISE & Ctrl+E & SBIE & CINTED & MPIE & SBgames & WIE & TOTAL \\
\hline 31 & 12 & 10 & 7 & 4 & 4 & 2 & 1 & 71 \\
\hline
\end{tabular}

Com o objetivo de organizar e facilitar a síntese dos dados, os estudos foram organizados de acordo com a fonte de publicação do ano mais atual (2020) para o mais antigo (2016). O gráfico 1 apresenta a distribuição dos artigos selecionados, incluídos por ano de publicação.

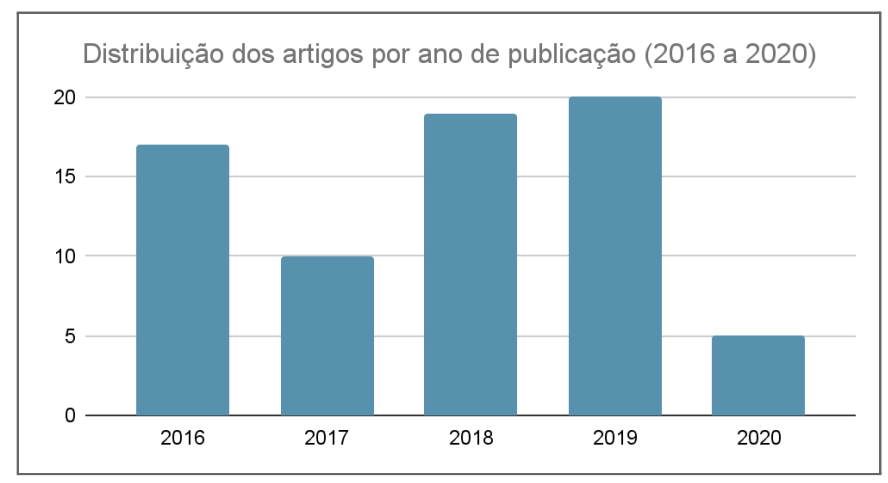

Gráfico 1: Artigos organizados por ano de publicação. Fonte: Os autores.

\subsection{Respostas às questões de pesquisa}

A primeira questão de pesquisa é: Quais técnicas são utilizadas para propor o ensino de programação para crianças? Essa questão ajudou a identificar quais foram as técnicas mais utilizadas para ensinar programação, algoritmos e proporcionar o desenvolvimento de habilidades do pensamento computacional na educação básica.

A técnica mais citada foi o desenvolvimento de jogos para ensinar programação, conforme a tabela 3. Crianças e jovens vivem imersos no mundo digital, por isso proporcionar um ensino que esteja vinculado com as atividades do dia a dia é fundamental para o desenvolvimento efetivo do conhecimento. A técnica de utilizar o desenvolvimento de jogos se tornou algo positivo e atrativo, por que os estudantes veem os jogos como entretenimento, onde é nítido que existe competitividade e engajamento para vencer. Dessa forma, pode-se utilizar na aula a competitividade de forma eficaz, para produzir um produto real onde os alunos irão precisar pensar em aspectos que tornem o desenvolvimento deles diferenciados dos demais. Ensinar programação com base no desenvolvimento de jogos é desafiar a criatividade e o potencial que cada estudante tem, para assim desenvolver um jogo do zero, iniciando a partir dos conceitos aprendidos em sala.

A segunda técnica mais citada foi a utilização de abordagens de programação através do pensamento computacional, onde os alunos podem iniciar a aprendizagem de programação a partir de pequenos algoritmos que precisam ser resolvidos. Assim como podem utilizar o pilar de decomposição para dividir uma tarefa em partes, antes de iniciar a programação visual, facilitando na organização das etapas a serem seguidas para obter o resultado final. 
A terceira técnica mais citada foi a utilização de storytelling como suporte para tornar a aula mais atrativa e compreensível, já que com essa técnica as problemáticas passam a ser vistas em forma de narrações, histórias que precisam ser lidas ou ouvidas, para pensar na elaboração de solução.

A quarta técnica mais citada foi o uso das práticas de robótica, algo que chama muito a atenção dos alunos, por proporcionar um momento de criação de dispositivos eletrônicos. Mesmo que muitas vezes sejam utilizadas ferramentas de simulação, existe o processo de montagem do dispositivo e o momento de programar sua função. Trabalhar o ensino e aprendizagem com o uso da robótica proporciona sempre um trabalho prático, que ajuda a tornar a aula mais atrativa.

A quinta técnica mais citada foi a utilização de oficinas práticas com conteúdos lúdicos, baseadas em desafios que são resolvidos com o desenvolvimento da programação de personagens, que precisam executar alguma função já definida e apresentada através dos conteúdos lúdicos, sendo: vídeos, histórias e entre outros.

A sexta técnica mais citada foi o ensino interdisciplinar, onde conceitos de programação podem ser introduzidos juntamente com conteúdos de outras disciplinas, como matemática, onde o aluno pode aprender sobre operadores relacionais e aritméticos programando funções matemáticas para a resolução de problemas.

Por fim, a última técnica citada foi a computação desplugada, onde o aluno passa a aprender conceitos de programação sem a utilização de nenhum dispositivo eletrônico. Essa prática é fundamental para escolas que não possuem laboratórios de computação.

Tabela 3. Técnicas de ensino identificadas.

\begin{tabular}{|l|c|}
\hline \multicolumn{1}{|c|}{ Técnicas citadas } & Quantidade \\
\hline Desenvolvimento de jogos pelos alunos. & 21 \\
\hline Abordagem através do pensamento computacional. & 14 \\
\hline Storytelling & 8 \\
\hline Práticas com o uso da robótica. & 8 \\
\hline Oficinas práticas com conteúdo lúdico & 6 \\
\hline Ensino interdisciplinar. & 5 \\
\hline Computação desplugada. & 3 \\
\hline
\end{tabular}

A segunda pergunta da pesquisa é: Quais são os objetivos do ensino de programação para crianças? As respostas obtidas para essa questão permitiram identificar que o ensino de programação busca proporcionar autonomia para que os alunos consigam identificar problemas desde muito cedo e possam elaborar soluções para resolvê-los da melhor forma possível. Na análise, houve quatro objetivos mais citados, que estão mostrados no gráfico 2 . 


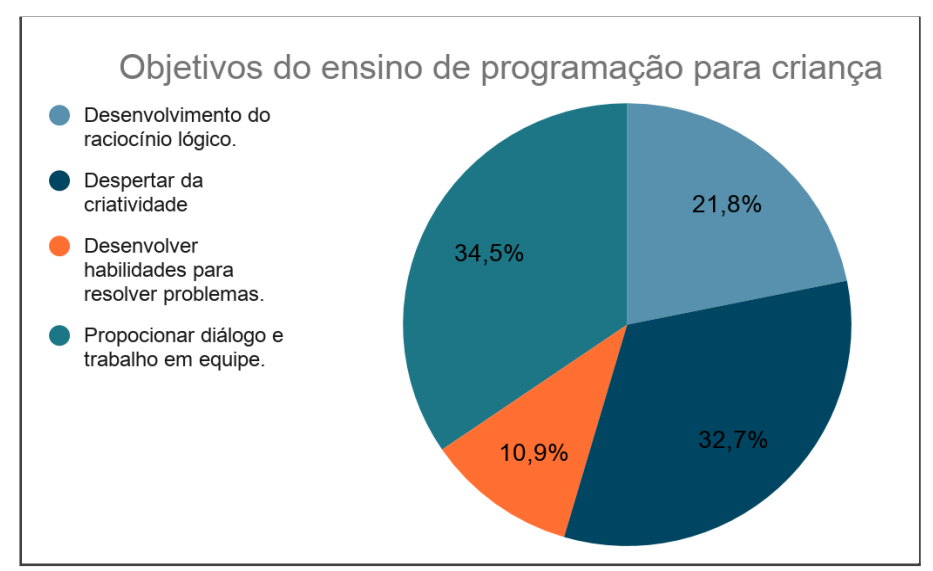

Gráfico 2. Objetivos mais citados. Fonte: Os autores.

Os aprendizados adquiridos com os conceitos de programação oportunizam o desenvolvimento de habilidades que contribuem não somente para o entendimento de softwares na área da tecnologia, mas também, para ter competências para realizar atividades do cotidiano. Pois, ter a capacidade de desenvolver algoritmos para resolver problemas é algo fundamental, e quando o problema passa a ser resolvido de forma criativa com diálogo construtivo é algo vantajoso e um conhecimento privilegiado para aqueles que o detêm.

A terceira pergunta de pesquisa é: Quais ferramentas educacionais são utilizadas? Essa questão permitiu identificar quais foram as linguagens e ambientes de programação utilizados para apoiar o ensino de programação, com destaque para a linguagem Scratch que foi a mais citada durante a análise, por conter uma interface intuitiva e proporcionar o desenvolvimento de jogos, animações e histórias. As linguagens e ambientes de programação mais citados são mostrados no Gráfico 3.

É preciso estabelecer algum critério para caracterizar uma ferramenta ideal para o ensino de programação na educação básica, analisando o quanto a ferramenta pode contribuir para um aprendizado eficaz. Para Macedo et al. (2005), um critério fundamental é a utilização de ferramentas lúdicas, que proporcionam para o ensino e aprendizagem as seguintes qualidades: tornam as tarefas prazerosas, são desafiadoras, possuem dimensão simbólica e não limitam as possibilidades.

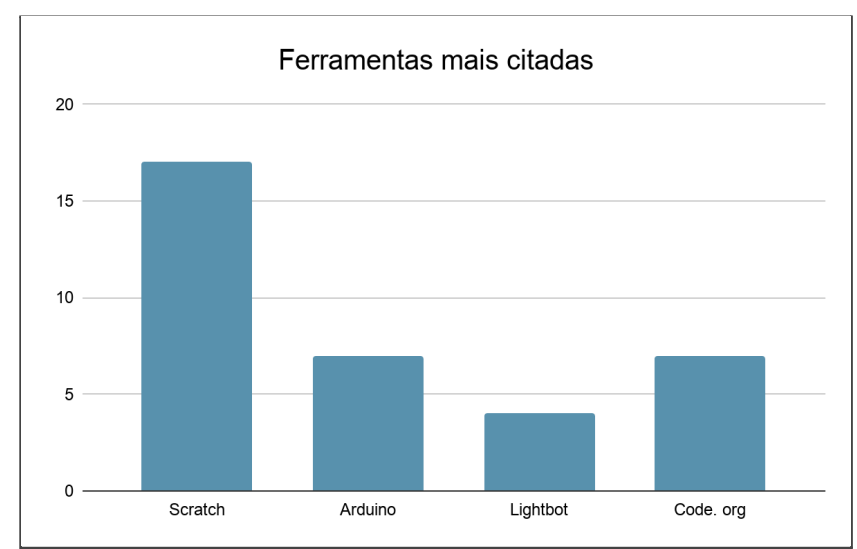

Gráfico 3. Ferramentas mais citadas na análise. Fonte: Os autores.

De acordo com a análise, o Scratch é a ferramenta mais citada por disponibilizar diferentes funções para os usuários, além de uma interface simples que contribui para o aprendizado das funções que cada bloco possui. Por ser uma ferramenta de programação em blocos robusta, é possível adquirir o aprendizado através de programações simples, voltadas para usuários iniciais, e também pode-se realizar programações mais elaboradas para a produção de animações ou jogos mais complexos. Já o Arduino foi a segunda ferramenta 
Anais do XXXII Simpósio Brasileiro de Informática na Educação (SBIE 2021)

mais citada por proporcionar uma experiência realista de robótica. O Arduino passou a ser bastante utilizado por ter elementos de baixo custo, e ainda por possuir uma ferramenta de simulação ${ }^{2}$ onde a programação e a montagem de peças virtuais podem ser testadas antes da produção final de forma física.

O code.org é um site que disponibiliza diversos jogos que geralmente são acessados por estudantes iniciantes, que possuem a pretensão de aprender conceitos básicos da programação, como estruturas de repetição e condição. $O$ site foi citado como a terceira ferramenta mais utilizada.

O jogo Lightbot (citada como a quarta ferramenta mais utilizada) é muito utilizado para o início do ensino de programação para crianças, pois se trata de uma ferramenta de simples compreensão que enfatiza o seguimento de algoritmos para cumprir as missões determinadas.

A quarta pergunta de pesquisa é: Como o pensamento computacional influencia no desenvolvimento do aprendizado em outras disciplinas? Na análise, quatro disciplinas foram citadas, onde a computação e seus conceitos contribuem para que ocorra engajamento nos conceitos e práticas. Pois, através da computação e do pensamento computacional, conceitos de outras disciplinas podem ser trabalhados de forma lúdica e divertida, facilitando o aprendizado de conceitos em que os alunos apresentam dificuldades. $\mathrm{O}$ gráfico 4 mostra as disciplinas mais citadas por fazer uso dos conceitos do pensamento computacional.

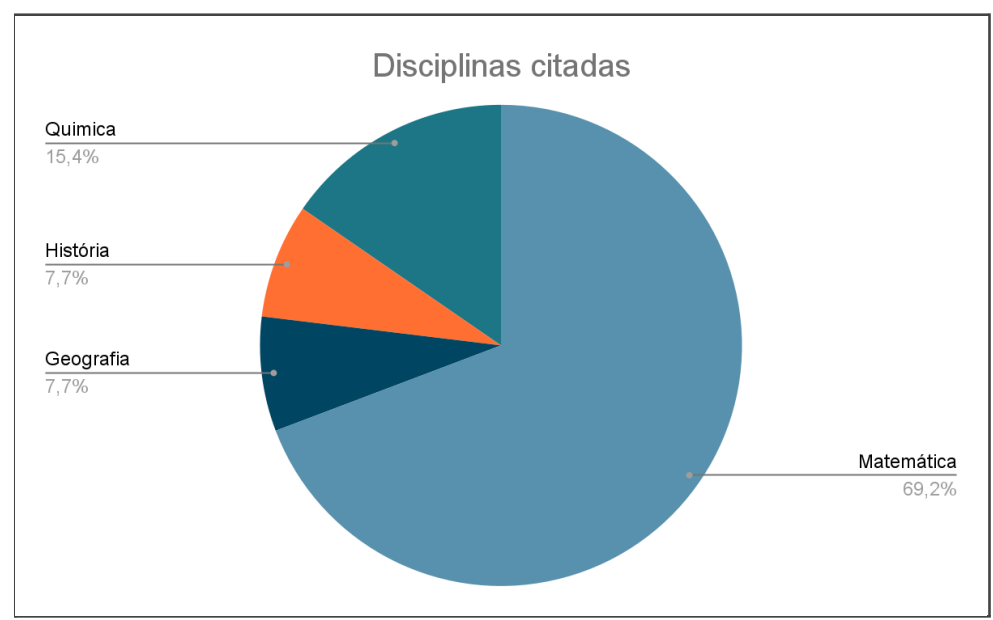

Gráfico 4. Disciplinas mais citadas na análise. Fonte: Os autores.

Alguns trabalhos analisados apresentam como os pilares do pensamento computacional podem ser utilizados no ensino de matemática, onde pode-se utilizar por exemplo o pilar de decomposição, para dividir um problemas em partes, na intenção que cada parte seja resolvida separadamente, para ao final existir uma resposta completa para o problema.

O pilar de algoritmo pode ser utilizado para diversas finalidades, como citado em alguns artigos analisados, onde pode-se utilizar algoritmos para executar uma sequência de passos que irão proporcionar o desenvolvimento de jogos, que possibilitem a disseminação de conhecimentos da área de ciências.

Através dos pilares de abstração e reconhecimento de padrões, os alunos podem adquirir habilidades para serem utilizadas em qualquer área do conhecimento, pois se trata de abstrair informações que não são relevantes em determinadas situações. E adquirir a facilidade de reconhecer padrões já vistos anteriormente, que podem contribuir para a construção de soluções mais eficientes para determinados problemas.

Para complementar os resultados descritos nesta seção, os dados dos respectivos

\footnotetext{
${ }^{2}$ Acesso para o programação de simulação do Arduino: https://www.tinkercad.com/
} 
trabalhos foram organizados em uma planilha ${ }^{3}$ onde é possível observar quais artigos foram selecionados para responder às perguntas de pesquisa.

\section{Considerações finais e trabalhos futuros}

Este artigo apresentou uma revisão da literatura com o objetivo de investigar questões relacionadas ao ensino de programação na educação básica e quais metodologias, ferramentas e estratégias são mais utilizadas neste processo. Durante a análise ficaram evidentes as diversas contribuições positivas que a aprendizagem de programação pode proporcionar, inclusive tornando os alunos mais aptos a resolverem problemas de outras disciplinas, através de conceitos computacionais.

Foi possível observar que a maioria dos trabalhos selecionados de acordo com os critérios estabelecidos teve o Scratch como ferramenta escolhida para abordar os conteúdos de lógica de programação. Também observaram-se diferentes estratégias utilizadas para tornar o ensino mais lúdico e próximo da realidade dos alunos.

A principal limitação deste estudo está relacionada ao processo de busca em que nos concentramos apenas em artigos de anais de conferências e publicados apenas na Língua Portuguesa. Essa decisão se deu devido a limitação de espaço para reportar os resultados neste artigo, mas uma busca incluindo novos artigos será conduzida. A segunda limitação foi buscar artigos apenas dos últimos cinco anos, porém o critério de definição de cinco anos anteriores, se deu pelo fato do ensino de programação para criança ter se tornado popular exatamente no decorrer desses anos. Por essa razão não foram listados trabalhos que poderiam ser relevantes, porém de anos anteriores.

Como trabalhos futuros, a intenção é conduzir estudos focados na utilização de storytelling como apoio fundamental para o ensino-aprendizagem de programação na educação básica, de modo a caracterizar as narrativas como uma das principais técnicas a serem utilizadas para possibilitar uma melhor compreensão e adaptação a conceitos iniciais da programação. Além disso, é viável acrescentar novas buscas realizadas em periódicos, com a finalidade de tornar o trabalho mais robusto e suprir uma das limitações citadas.

\section{Referências}

AN. D.Y; SILVA. C. D; RIBEIRO. D. M.G; ROCHA. P. B. R.; MALTINTI. C.; NUNES. V. B.; FÁVERO, R.. Digita - um Jogo Educativo de Apoio ao Processo de Alfabetização Infantil. In: Anais do Simpósio Brasileiro de Informática na Educação, Vol. 24. No 1. 2013.

ARIMOTO, Maurício; OLIVEIRA, Weldrey. Dificuldades no Processo de Aprendizagem de Programação de Computadores: um Survey com Estudantes de Cursos da Área de Computação. In: WORKSHOP SOBRE EDUCAÇÃO EM COMPUTAÇÃO (WEI), 27. 2019, Belém. Porto Alegre: Sociedade Brasileira de Computação, 2019 . $\quad$ p. 244-254.

D. Oliveira; S. Ferreira; H. Celestino; S. Ferreira; P. Abrantes. Uma proposta de ensino-aprendizagem de programação utilizando robótica educativa e storytelling. In: II Congresso Internacional TIC de Educação, Lisboa. 2012. p. 10.

DOMINGOS, Adenil Alfeu. Storytelling: Evolução, Novas Tecnologias e Mídia. Intercom Sociedade Brasileira de Estudos Interdisciplinares da Comunicação, Curitiba, set. 2009.

FERRI, Juliana. Ensino de linguagem de programação na educação básica:uma proposta de sequência didática para desenvolver o pensamento computacional. universidade estadual do norte do paraná.

\footnotetext{
${ }^{3}$ Acesso para os dados dos respectivos trabalhos: encurtador.com.br/btOX2
} 
X Congresso Brasileiro de Informática na Educação (CBIE 2021)

Anais do XXXII Simpósio Brasileiro de Informática na Educação (SBIE 2021)

FERRI, Juliana; ROSA, Selma dos Santos. Como o Ensino de Programação de Computadores Pode Contribuir Com a Construção de Conhecimento na Educação Básica Uma Revisão Sistemática da Literatura. CINTED-UFRG, 2016.

FERREIRA, Luísa S. Santos, Sylvana Karla S. L., Bonfim, Cristiane Jorge L. Pensamento Computacional e Programação Scratch: uma revisão de literatura do SBIE. Instituto Federal de Brasília, 2021.

GOMES, Marcos César Pires. Os benefícios do ensino de linguagem de programação no currículo regular, 2015.

KELLEHER, C. and Pausch, R. (2005). Lowering the barriers to programming: A taxonomy of programming environments and languages for novice programmers. ACM Computing Surveys (CSUR), 37(2):83-137. Publisher: ACM New York, NY, USA.

Kitchenham, B.; Charters, S. (2007) Guidelines for performing Systematic Literature Reviews in Software Engineering. In: Technical report, Ver. 2.3 EBSE Technical Report. EBSE.

MACEDO, L., PETTY, A. L. S., PASSOS, N. C. (2005). Os jogos e o lúdico na aprendizagem escolar.

MEDEIROS, Rodrigo Pessoa; FALCÃO, Taciana Pontual; RAMALHO, Geber Lisboa. Ensino e Aprendizagem de Introdução à Programação no Ensino Superior Brasileiro: Revisão Sistemática da Literatura. In: WORKSHOP SOBRE EDUCAÇÃO EM COMPUTAÇÃO (WEI). Cuiabá. Porto Alegre: Sociedade Brasileira de Computação, 2020 .

ORO, Neuza et al. Olimpíada de Programação de Computadores para Estudantes do Ensino Fundamental: A interdisciplinaridade por meio do Software Scratch. In: Anais do Workshop de Informática na Escola. 2015. p. 102.

PALACIOS, Fernando; TERENZZO, Martha. O Guia Completo do Storytelling. Alta Books Editora, 2016.

S. M. Torres; A.L.L. Tettamanzy. Contação de histórias: resgate da memória e estímulo à imaginação. Nau literária. Porto Alegre, RS. Vol. 4, n. 1 (jan./jun. 2008), p. 1-8, 2008.

SANTOS, R. P.; Costa, H. A. X. (2006). Análise de metodologias e ambientes de ensino para algoritmos, estrutura de dados e programação aos iniciantes em Computação e Informática. INFOCOMP Journal of Computer Science, 5(1):41-50.

SELBY, C.; woollard, J. Computational thinking: the developing definition, 2013.

SERRANO, F. Geração Geek. Revista Exame Informática. São Paulo: Editora Abril, 2014.

SILVA, Jailson Cunha da. Ensino de Programação para alunos do Ensino Básico: Um levantamento das pesquisas realizadas no Brasil. Universidade Federal da Paraíba (UFPB) Campus IV -Rua da Mangueira, s/n, Companhia de Tecidos Rio Tinto, 2017.

SOUZA, Draylson Micael; BATISTA, Marisa Helena da Silva; BARBOSA, Ellen Francine. Problemas e Dificuldades no Ensino e na Aprendizagem de Programação: Um Mapeamento Sistemático. Revista Brasileira de Informática na Educação, Volume 24, Número 1, 2016.

WEINTROP, D., Shepherd, D. C., Francis, P., and Franklin, D. (2017). Blockly goes to work: Block-based programming for industrial robots. In 2017 IEEE Blocks and Beyond Workshop (B B), pages 29-36.

WING, J. Computational thinking. Communications of the acm, v. 49, n. 3, 2006, p. 33-35. 